\title{
Associations between renalase concentration and the occurrence of selected diseases
}

\author{
Justyna Czubilińska-Łada ${ }^{\circledR 1}$, Aleksandra Gliwińska ${ }^{\circledR 2}$, Andrzej Badeński ${ }^{\circledR 2}$, Maria Szczepańska ${ }^{\circledR 3}$ \\ ${ }^{1}$ Department of Neonatal Intensive Care and Neonatal Pathology, Faculty of Medical Sciences in Zabrze, Medical University \\ of Silesia in Katowice, Poland \\ ${ }^{2}$ Paediatric Nephrology Ward with Dialysis Division for Children, Public Clinical Hospital No. 1 in Zabrze, Poland \\ ${ }^{3}$ Department of Paediatrics, Faculty of Medical Sciences in Zabrze, Medical University of Silesia in Katowice, Poland
}

\begin{abstract}
Renalase is a recently identified flavoprotein oxidase, secreted mainly by the kidneys, which takes part in the degradation of catecholamines. The catecholamine inactivating effect results in the modulation of the sympathetic system tension and, consequently, in a decrease of blood pressure, myocardial contractility, heart rate, and vascular tone. Besides its enzymatic capacity, renalase shows cytoprotective properties by activating mitogen-activated protein kinase (MAPK) pathway. Several single nucleotide polymorphisms (SNPs) of the renalase gene have been identified, of which the most widely studied in relation to the development of selected diseases are rs2296545, rs10887800, and rs2576178. Numerous publications prove the contribution of renalase to the occurrence of cardiovascular diseases, kidney diseases, ischaemic stroke, diabetes type 1 and 2, as well as female infertility and schizophrenia. Further extended research into the various mechanisms of renalase activity may result in the use of this oxidase or its analogues as a therapeutic and/or diagnostic tool. (Endokrynol Pol 2020; 71 (4): 334-342)
\end{abstract}

Key words: renalase; catecholamines; cardiovascular diseases; kidney diseases

\section{Introduction}

Renalase is a flavoprotein oxidase whose major sources are renal proximal tubules. To a lesser degree, it is also expressed in the heart, skeletal muscles, endothelium, small intestine, adipose tissue, brain, liver, and reproductive/steroidogenic system. Initially only four, and currently as many as seven isoforms of human renalase are described ( $\mathrm{h}$ renalase $1-7$ ). The main and only isoform that has so far been isolated from blood and urine is h-renalase- 1 . This molecule is composed of 342 amino acids, and its molecular weight reaches $37.85 \mathrm{kDa}$ [1-3]. Renalase has a similar amino acid identity as monoamine oxidase A (MAO-A) at a level of $13.2 \%$. Both of these molecules inactivate catecholamines, although renalase is the only identified amine oxidase functioning extracellularly. The inactivation of catecholamines can be achieved due to the presence of cofactors flavin adenine dinucleotide (FAD) and nicotinamide adenine dinucleotide (NAD), and it occurs with the greatest affinity for dopamine, followed by adrenaline and ultimately noradrenaline $[1,4,5]$. Firstly, secreted into the blood as an inactive form of prorenalase, it rapidly evolves into renalase, under the influence of excess catecholamines and the rise in blood pressure. The main function of activated renalase is to modulate blood pressure and heart rate by affecting fluctuations in catecholamine levels in peripheral blood $[6,7]$. In recent years, a group of scientists represented by Baupre et al. published a study presenting an alternative metabolic pathway for renalase, in which it acts as an $\alpha-\mathrm{NAD}(\mathrm{P}) \mathrm{H}$ oxidase/anomerase. The described reaction consists of a rapid reduction of the renalase flavin cofactor thanks to low NADH and NADH equilibrium concentrations, followed by the reaction of the reduced cofactor with molecular oxygen. This reaction produces hydrogen peroxide and nicotinamide dinucleotide in the $\beta$-form, and this entire process is at least two times faster than any reaction previously proposed with catechol neurotransmitters $[8,9]$. Independently of its enzymatic functions, renalase has the properties of cytokine, which, as a result of reacting with plasma membrane receptor PMCA4b, activates the mitogen-activated protein kinase (MAPK) pathway. The consequence of triggering this cascade is a cytoprotective, anti-inflammatory effect and protection from hypoxia and apoptosis $[10,11]$. Different kinds of renalase activity make it necessary to search for its participation in the pathophysiology of diseases as well as potential therapeutic and diagnostic effects. 


\section{Renalase gene polymorphism}

Nowadays, genome-wide association studies (GWAS) have become a well-established method in the exploration of polymorphisms of particular genes contributing to the development of many complex diseases. The human renalase gene (RNLS) is located on chromosome $10 \mathrm{q} 23.33$ and consists of $\sim 310 \mathrm{Kbp}$ with 10 exons [12]. Genetic analysis revealed many single nucleotide polymorphisms (SNPs) of the renalase gene associated with a wide range of diseases. The greatest amount of evidence is mainly based on the SNPs rs2296545, rs10887800, and rs2576178 connected with different cardiovascular disorders, kidney diseases, diabetes type 1 and 2, ischaemic stroke, and others (Tab. 1) [10, 12].

\section{Coronary artery disease}

Understanding the renalase impact on oscillations of catecholamine levels explains its role in the regulation of blood pressure, myocardial contraction, heart rate, and vascular tone [14]. Coronary artery disease is first among cardiovascular diseases in terms of occurrence and mortality. The aetiology of these illnesses is multifactorial, including congenital predispositions, lifestyle, and environmental factors. The correlation between renalase and the prevalence of coronary artery disease (CAD) is widely described. He et al. conducted a study on CAD patients divided into groups according to the number of occupied coronary artery branches. Not only were significantly increased levels of renalase in the control group revealed, but also dependencies of the renalase concentration on the severity of the disease [15]. The relationship between the polymorphism of the renalase gene (rs2576178 and rs10887800) and the occurrence of CAD has been demonstrated several times [16]. The research performed by Li et al. compared renalase SNPs among patients with hypertension and accompanying CAD versus patients with hypertension only [17]. Also, Stec et al. confirmed the connection between the renalase gene and CAD by performing a study of patients on haemodialysis [18].

\section{Primary hypertension}

Primary hypertension is a complex disease, and the literature describes many mechanisms that may contribute to the development of this disease or intensify its course. The factors involved in the pathophysiology of hypertension development include increased salt intake, abnormal regulation of the renin-angiotensin-aldosterone system (RAAS), and hyperactivation of the sympathetic system [19-21]. The low level of renalase can contribute to the development of primary
Table 1. The most studied single nucleotide polymorphisms (SNPs) of renalase gene related with elevated risk of selected diseases

\begin{tabular}{lll}
\hline $\begin{array}{l}\text { Single nucleotide } \\
\text { polymorphisms }\end{array}$ & Disease & References \\
\hline rs2296545 & Hypertension & {$[25]$} \\
\hline & Coronary artery disease & {$[18]$} \\
& Pregnancy-induced & {$[26]$} \\
hypertension & {$[27-28]$} \\
& Preeclampsia & {$[40-42]$} \\
& Ischaemic stroke & {$[47]$} \\
& Women infertility & {$[16,17]$} \\
& Coronary artery disease & {$[25]$} \\
& Hypertension & {$[26]$} \\
& Pregnancy-induced & {$[28]$} \\
& hypertension & {$[42]$} \\
\hline rs10509540 & Preeclampsia & {$[47]$} \\
\hline & Ischemic stroke & {$[44]$} \\
\hline
\end{tabular}

hypertension in the mechanism of hyperactivation of the sympathetic nervous system. Maciorkowska et al. conducted experimental studies on patients with primary hypertension, whose concentrations of renalase, vascular adhesion protein 1 (VAP-1), and catecholamines in serum were measured. The concentrations of renalase and VAP-1 were significantly higher in the study group compared to healthy individuals. Moreover, a correlation between renalase and norepinephrine concentrations was observed as well as a correlation between renalase concentrations and both systolic and diastolic ambulatory blood pressure measurements [22]. Opposite results were obtained by Wybraniec et al., who, from 50 patients after surgical aortic coarctation correction and 50 healthy patients, selected those with arterial hypertension for further investigation. In both groups renalase concentrations were significantly lower among patients with hypertension, but what distinguishes these studies from the others presented above is the fact that renalase was determined in plasma instead of serum [23]. Similar findings were presented by Schlaich et al., showing a decreased concentration of plasma renalase among patients with resistant hypertension compared to healthy individuals. The difference between these studies and the others is the method for determining the concentration of the renalase - western blot technique instead of enzyme-like immunosorbent assay (ELISA) [24]. In spite of experimental studies determining the levels of renalase in serum or plasma of peripheral blood plasma in patients with hypertension, genetic 
relationships between renalase and essential hypertension have been determined. Zhao et al. presented the association between single nucleotide polymorphisms in the renalase gene (rs2296545 and rs2576178) and the occurrence of essential hypertension among the Han Chinese population [25]. It seems to be certain that renalase and hypertension are related, but it is not clear whether abnormal renalase concentration is a factor predisposing to hypertension or is its result.

\section{Hypertension during pregnancy}

Hypertension also affects the group of pregnant women and constitutes $2-8 \%$ of all pregnancy complications. Several studies proved the connection between the polymorphism of the renalase genes (rs 10887800 and rs2576178) and the occurrence of elevated pressure in the course of pre-eclampsia (PE) and pregnancy-induced hypertension (PIH). Elsethoy et al. submitted a study of women suffering from PIH, which revealed that the distribution of both genotypes mentioned above was significantly higher in the study group comparing to the controls. The research also presents graduating values of systolic and diastolic blood pressures according to the genotype dominance [26]. Similar studies carried out by Bagci et al. concerned a group women with PE, not only showing the polymorphism of the rs108878800 renalase gene, but also confirming a significant statistical differentiation of systolic and diastolic pressure depending on the genotype [27]. Teimoori et al. demonstrated opposite results by studying SNPs of a renalase gene in women with PE. They showed that the combination of rs10887800 GG and rs2576178 GG genotypes significantly increased the risk of pre-eclampsia, although the polymorphisms themselves were not significantly more frequent in the study group than in the controls [28]. Serum renalase determinations in pre-eclampsia, hypertension, and healthy pregnant women were performed by Jamil et al. without significant differences in renalase concentrations between these three groups [29]. However, two independent scientific groups represented by Niadany et al. and Yilmas et al. presented significantly lower renalase concentrations in PE women compared to healthy pregnant women [30, 31]. In conclusion, it has been repeatedly proven that the polymorphism of the renalase gene is associated with the occurrence of hypertensive conditions in pregnant women and can be considered as a genetic risk factor of these conditions.

\section{Chronic kidney disease}

The links between renalase and kidney diseases are widely described in the literature. Chronic kidney disease (CKD) is the most widely described kidney disorder associated with renalase levels in both adults and children. Several studies have proven that the concentrations of renalase in serum are significantly higher in patients with CKD than in the control group and elevated with the increasing stage of renal failure. A study among a group of patients undergoing haemodialysis was carried out by Zbroch et al., who documented significantly higher renalase concentrations in the study group than in healthy individuals. The level of renalase concentration was not related to arterial pressure, heart rate, time of haemodialysis, type of hypotensive treatment, or residual kidney function [32]. Slightly later, research involving patients undergoing peritoneal dialysis, besides the haemodialysis group, was presented. In addition to the renalase, dopamine and norepinephrine concentrations were also determined in all studied groups; however, no correlation between all these substances was proven. Among the patients undergoing haemodialysis, patients with concomitant coronary artery disease were identified and diagnosed with cardiac death. In this group renalase concentration was significantly higher than in other haemodialyzed patients, which is a hint to deepen the pathophysiology of renalase in patients with CKD and CAD [33]. Subsequent studies confirmed higher renalase concentrations among patients with CKD and a significant correlation between renalase levels and the renal injury markers creatinine and urea [34, 35]. Similar results are presented in a study group that included children suffering from CKD [36]. Wiśniewska et al. presented corresponding conclusions concerning the group of patients with CKD, among whom serum renalase concentration was significantly higher than in the control group. Also, the measurement of renalase in erythrocytes was performed and showed a decrease in renalase and an increase in urea concentration in the control group [37]. All of these studies used the ELISA technique to determine plasma renalase concentration. Research involving western blot technique presented contrasting results, in which renalase concentrations were significantly lower in patients with CKD than in the controls. Despite the different techniques, a relationship between the CKD stage and the renalase concentration was also observed [1,7]. The polymorphism of the renalase gene associated with kidney diseases was investigated in patients after allogeneic kidney transplants; however, no significant connection with renal transplant function was established [38].

\section{Ischaemic stroke}

The fact that the expression of renalase also occurs in endothelium cells raises the suspicion that this enzyme 
may be involved in the pathogenesis of diseases that are caused by endothelial dysfunction [39]. This broad disease spectrum includes disorders associated with atherosclerotic plaque formation such as an ischaemic stroke. The association of the rs10887800 gene polymorphism with ischaemic stroke was reported in several studies. Firstly, Buraczyńska et al. performed tests on type 2 diabetes patients and proved the presence of SNP rs108887800 in the renalase gene in hypertensive patients with stroke. This finding was extended by examining a separate cohort of stroke patients free of diabetes. The association of the renalase gene with an increased risk of ischaemic stroke was confirmed in patients with and without type 2 diabetes [40]. In a study conducted by Li et al. a cohort of 212 ischaemic stroke patients and 244 controls were divided into groups according to the stage of stenosis of intracranial cerebral atherosclerotic vascular stenosis. The severity of the disease was assessed by imaging exams. It was shown that rs10887800 is connected with intracranial cerebral atherosclerotic vascular stenosis in ischaemic stroke patients [41]. At a similar time, Zhang et al. published their research in which they drew similar conclusions. In their study, 503 patients with ischaemic stroke were divided into groups due to complications and accompanying diseases such as hypertension and diabetes. A strong correlation between both rs10887800 and rs2576178 SNPs and ischaemic stroke was demonstrated in both hypertensive and non-hypertensive patients [42]. The connection between renalase and ischaemic stroke was also raised in the pilot study performed by Malyszko et al. on a group of haemodialyzed patients. The serum renalase concentration was significantly lower in patients with a history of stroke. All the above studies show a link between renalase and ischaemic stroke, but the aetiology of this phenomenon is still unexplained [43].

\section{Diabetes type 1 and 2}

The risk of developing type 1 diabetes increases with the presence of common genetic loci. This knowledge has established the autoimmune basis of the disease. Genome-wide association studies present approximately 42 loci that affect the risk of type 1 diabetes and 70 regions with suggestive evidence. Among this group, there is a region rs10509540 located in the renalase gene, which makes it possible to link type 1 diabetes and renalase, but this mechanism needs further investigation [44]. The relationship between renalase and type 2 diabetes mellitus (T2DM) was studied by Wang et al., who performed serum renalase and catecholamine assays in 75 T2DM patients and 13 healthy individuals. Furthermore, in addition to significantly higher concentrations of renalase and catecholamines in the control group, it was demonstrated that the renalase/catecholamine ratio correlated strongly with the occurrence of insulin resistance [45].

\section{Female infertility}

The growing number of infertile couples around the world, currently reaching $10-15 \%$, is leading to the search for its causes in genetics [46]. The research performed by Fatima et al. to discover a polymorphism of the renalase gene among infertile women included 508 fertile and 164 women who were infertile due to various reasons. The study proved a significant correlation of SNPs rs2576178 and rs10887800 with female infertility of unknown cause and with infertility caused by polycystic ovarian syndrome. Scientists have hypothesised that SNPs in the renalase gene may interfere with the hormonal hypothalamic-pituitary-associated ovarian axis by affecting the degradation of catecholamines and increasing the number of stress hormones [47]. The link between renalase and the reproductive system is supported by the fact that Zhou et al. proved that the gonads and adrenal cortex are a source of renalase and suggested that it may affect fertility [3].

\section{Schizophrenia}

Catak et al. worked on a new direction of research on renalase, performing an assessment of the concentration of renalase and catecholamines among patients with schizophrenia. The speculations that renalase may be involved in the development of schizophrenia are justified by the fact that dopamine is involved in its pathogenesis. The study group showed statistically significantly lower renalase concentration and higher dopamine concentration compared to the controls [48]. Also, Hennebry et al. proved the presence of renalase in the peripheral nerves, hypothalamus, and pituitary gland and suggested that it could be involved in regulation of neurotransmitter levels in the brain [2]. On the basis of these results, there is a suspicion that renalase may be involved in the development of many psychiatric and neurological diseases, which requires further investigation.

\section{Renalase in children}

Publications concerning the determination of renalase concentrations in children are relatively limited so far. The majority of studies regarding adolescents involve nephrological patients; however, until now no renalase determinations have been performed in neonates. Two studies conducted by the group of Skrzypczyk et al. 
include children with glomerular kidney diseases (2017) and with chronic kidney diseases (2019). Although there was no significant difference between the renalase concentration in glomerulopathy patients and the control group, a correlation of renalase with daily proteinuria as well as with age, systolic and diastolic central blood pressure, eGFR, cholesterol, triglycerides, and pulse wave velocity was found. In a study on CKD patients, the results of renalase determinations were similar to those of most studies in the adult population. In the study group a higher concentration of renalase was found than in healthy individuals. Furthermore, a correlation between renalase and CKD stage and negative correlation with eGFR were observed. The authors observed significantly decreasing renalase concentrations with age among healthy patients, whereas there is no correlation between renalase and age in the study group [36, 49]. Taranta-Janusz et al. performed studies on paediatric patients with solitary functioning kidney and discovered lower serum and urine renalase concentrations in patients in the study group [50]. The study conducted among teenage hypertensive patients presented by Lemiesz et al. showed significantly higher renalase concentrations among the study group compared to healthy individuals. Furthermore, a significant correlation was found between the levels of renalase and uric acid and between renalase levels and measurements of systolic and diastolic blood pressure [51]. Rybi-Szumińska et al. conducted a study to establish urine renalase reference values in the paediatric population. Patients were divided into age groups in three-year intervals, which allowed them to observe a significantly higher renalase/creatinine ratio among patients less than three years old compared to older groups [52]. The fluctuation of renalase concentrations in the child population requires further evaluation. The number of studies among children is negligible; moreover, most of them are limited due to the small number of samples and low diversity among the study groups.

\section{Animal models}

Until now, many studies prosecuting animal experiments confirmed that the application of renalase triggers the decrease of blood pressure, myocardial contractility, and heart rate via regulating plasma catecholamine levels $[1,53,54]$. Renalase knockout mice developed hypertension and have more severe renal and cardiac ischaemia. Moreover, administrating recombinant renalase in wild-type mice limits ischaemic kidney injury by decreasing renal tubular necrosis, apoptosis, and inflammation [55, 56]. Cytoprotective properties of renalase have been demonstrated during an experiment with rats after subtotal (5/6) nephrec- tomy. Systemic delivery of renalase in the trial group prevented kidney injury and cardiac remodelling [14]. The impact of salt-intake on renalase expression in kidneys was confirmed by two independent experiments using Dahl salt-sensitive rats and Sprague-Dawley rats. Both investigations report that high salt intake inhibits the renal secretion of renalase. The influence of the renin-angiotensin-aldosterone system was excluded in this phenomenon $[57,58]$. An impact on perfusion of kidneys was validated in an animal model of rats presented by Gu et al., with unilateral renal artery stenosis and other groups with infarction-induced heart failure. In both groups diminished renal blood flow led to the reduced renal expression of renalase [59].

\section{Summary}

Knowledge about the function and properties of renalase as an enzyme and as a cytokine is constantly expanding. More and more organs and tissues in which renalase is produced or secreted into peripheral blood are being discovered. The association of renalase with various pathologies has been proven repeatedly and may provide a background for understanding the pathomechanism of its functioning. Determination of renalase concentration in the course of various chronic diseases has been evaluated in numerous studies (Tab. 2). Apart from learning the genetic connection of the renalase gene with diverse diseases and its influence on the course of these disorders, its therapeutic potential was also investigated. So far, numerous tests have been carried out on animal models using recombinant renalase. Due to the discovered properties of renalase based on catecholamine degradation and cytoprotective effects, it seems that therapeutic or diagnostic use of renalase may be valuable especially in states related to increased activity of the sympathetic system.

Further work should also be focused on better standardisation of the method for determining renalase concentration. Variations in the usage of different measurement methods are an important part of the scientific discussion on the interpretation of renalase concentration in peripheral blood serum. Increased renalase concentrations in ELISA tests may result from cross-reaction detection of nonbiologically inactive enzyme fragments accumulating in advanced kidney disease. On the other hand, it is worth mentioning that western blot studies also need to be refined, because they used measurement of MAO activity and extrapolated to renalase activity, while the similarity of the two substances is low [60, 61].

In conclusion, renalase plays a major role in the regulation of cardiovascular and renal systems via metabolising catecholamines and adjustment of the 


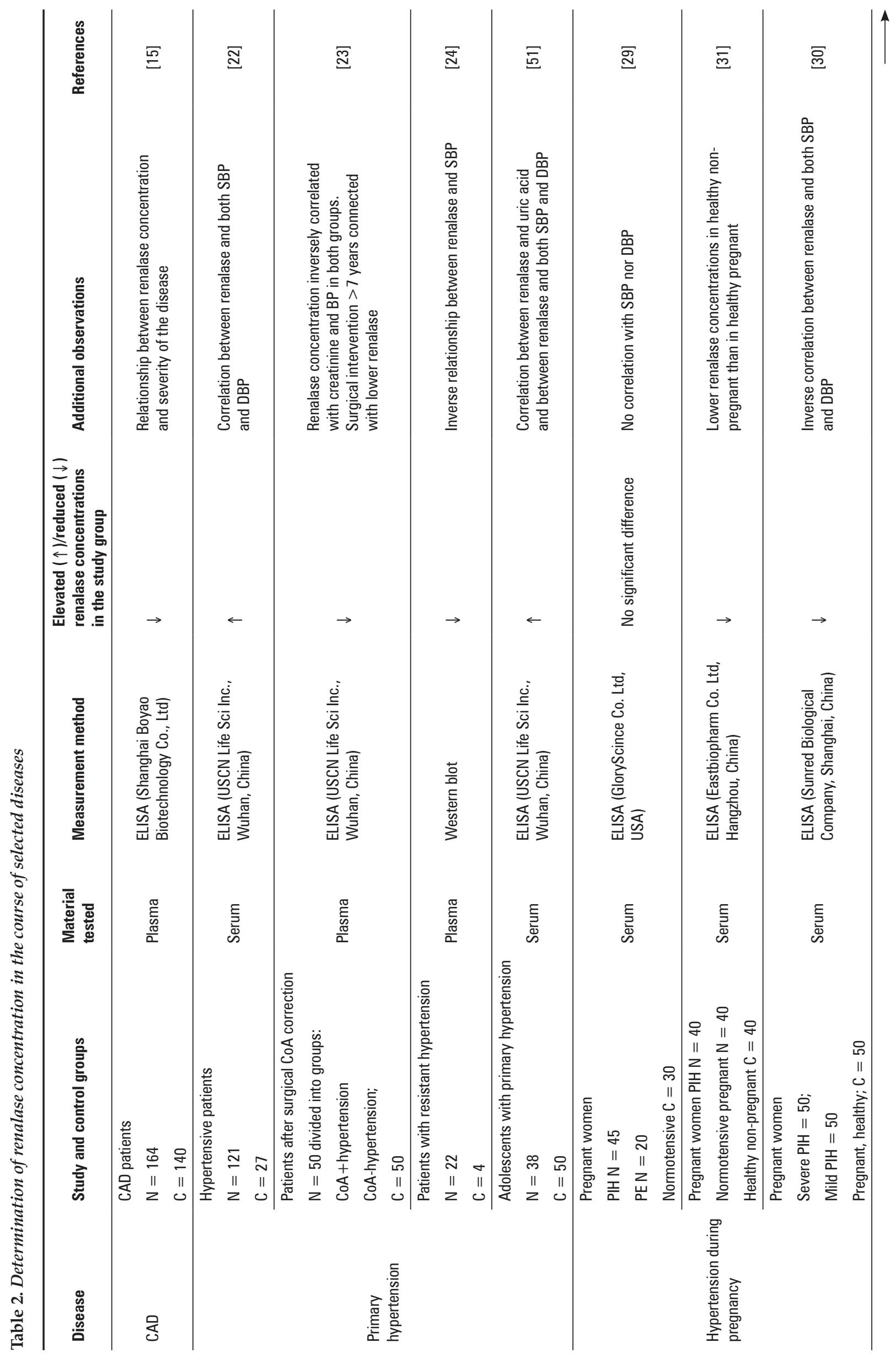




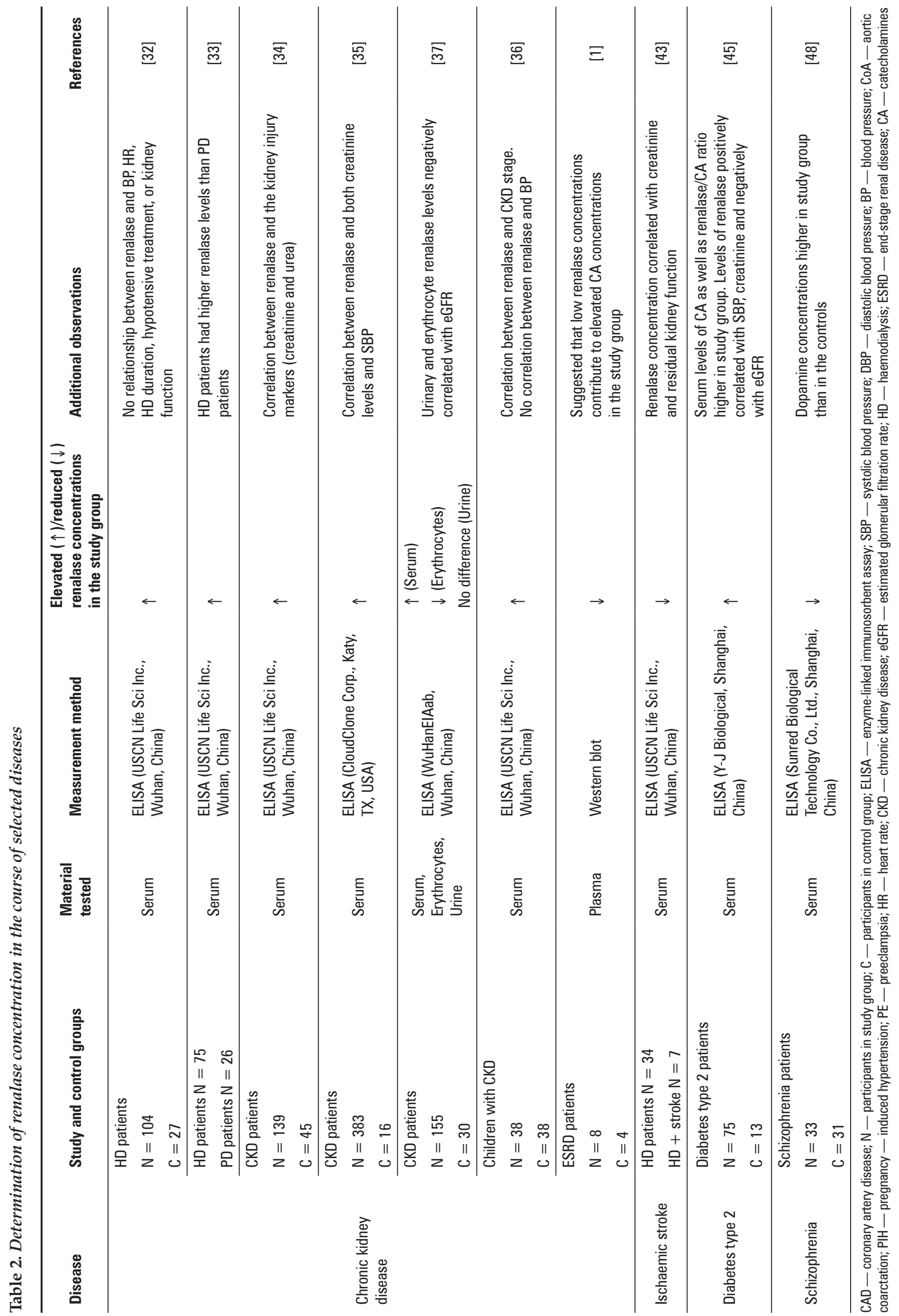


sympathetic nervous system. Understanding its signalling properties, independent of the enzymatic function, is an important step towards explaining the background of its cytoprotective and anti-inflammatory activity. The usage of recombinant renalase or its analogues is considered as a new possible therapeutic option.

\section{References}

1. $\mathrm{Xu} \mathrm{J}, \mathrm{Li} \mathrm{G}$, Wang P, et al. Renalase is a novel, soluble monoamine oxidase that regulates cardiac function and blood pressure. J Clin Invest. 2005; 115(5): 1275-1280, doi: 10.1172/JCI24066, indexed in Pubmed: 15841207.

2. Hennebry SC, Eikelis N, Socratous F, et al. Renalase, a novel soluble FAD-dependent protein, is synthesized in the brain and periphera nerves. Mol Psychiatry. 2010; 15(3): 234-236, doi: 10.1038/mp.2009.74, indexed in Pubmed: 20168325.

3. Zhou M, Liang T, Wang Y, et al. Expression and tissue localization of renalase, a novel soluble FAD-dependent protein, in reproductive/steroidogenic systems. Mol Biol Rep. 2013; 40(6): 3987-3994 doi: 10.1007/s11033-012-2476-0, indexed in Pubmed: 23271136

4. Xu J, Desir GV. Renalase, a new renal hormone: its role in health and disease. Curr Opin Nephrol Hypertens. 2007; 16(4): 373-378 doi: 10.1097/MNH.0b013e3281bd8877, indexed in Pubmed: 17565281.

5. Wang F, Xing T, Li J, et al. Renalase's expression and distribution in renal tissue and cells. PLoS One. 2012; 7(10): e46442, doi: 10.1371/journal. pone.0046442, indexed in Pubmed: 23056310.

6. Desir GV. Renalase deficiency in chronic kidney disease, and its contribution to hypertension and cardiovascular disease. Curr Opin Nephrol Hypertens. 2008; 17(2): 181-185, doi: 10.1097/MNH.0b013e3282f521ba, indexed in Pubmed: 18277152.

7. Li G, Xu J, Wang P, et al. Catecholamines regulate the activity, secretion, and synthesis of renalase. Circulation. 2008; 117(10): 1277-1282, doi: 10.1161/CIRCULATIONAHA.107.732032, indexed in Pubmed: 18299506.

8. Beaupre BA, Carmichael BR, Hoag MR, et al. Renalase is an $\alpha-\mathrm{NAD}(\mathrm{P})$ H oxidase/anomerase. J Am Chem Soc. 2013; 135(37): 13980-13987, doi: 10.1021/ja407384h, indexed in Pubmed: 23964689.

9. Moran GR, Hoag MR. The enzyme: Renalase. Arch Biochem Biophys. 2017; 632: 66-76, doi: 10.1016/j.abb.2017.05.015, indexed in Pubmed: 28558965.

10. Guo X, Wang L, Velazquez H, et al. Renalase: its role as a cytokine, and an update on its association with type 1 diabetes and ischemic stroke. Curr Opin Nephrol Hypertens. 2014; 23(5): 513-518, doi: 10.1097/MNH.0000000000000044, indexed in Pubmed: 24992568

11. Wang $\mathrm{L}$, Velazquez $\mathrm{H}$, Chang J, et al. Identification of a receptor for extracellular renalase. PLoS One. 2015; 10(4): e0122932, doi: 10.1371/journal. pone.0122932, indexed in Pubmed: 25906147.

12. Desir GV. Regulation of blood pressure and cardiovascular function by renalase. Kidney Int. 2009; 76(4): 366-370, doi: 10.1038/ki.2009.169, indexed in Pubmed: 19471322.

13. Lv YB, Wang Y, Ma WG, et al. Association of Renalase SNPs rs2296545 and rs2576178 with the Risk of Hypertension: A Meta-Analysis. PLoS One. 2016; 11(7): e0158880, doi: 10.1371/journal.pone.0158880, indexed in Pubmed: 27434211.

14. Akbari $\mathrm{H}$, Asadikaram $\mathrm{G}$, Vakili S, et al. Atorvastatin and losartan may upregulate renalase activity in hypertension but not coronary artery diseases: The role of gene polymorphism. J Cell Biochem. 2019; 120(6) 9159-9171, doi: 10.1002/jcb.28191, indexed in Pubmed: 30548657.

15. He B, Hao J, Sheng W, et al. Correlation between plasma renalase level and coronary artery disease. Pak J Med Sci. 2014; 30(5): 863-967, doi: 10.12669/pjms.305.5286, indexed in Pubmed: 25225499.

16. $\mathrm{Hu} \mathrm{N}$, Wang J, Hu P, et al. Investigation of Renalase gene rs 2576178 polymorphism in patients with coronary artery disease. Biosci Rep. 2018; 38(5), doi: 10.1042/BSR20180839, indexed in Pubmed: 30181378.

17. Li X, Jiang $\mathrm{W}, \mathrm{Li} \mathrm{L}$, et al. Renalase gene polymorphism in patients with hypertension and concomitant coronary heart disease. Kidney Blood Press Res. 2014; 39(1): 9-16, doi: 10.1159/000355771, indexed in Pubmed: 24821235.

18. Stec A, Ksiazek A, Buraczynska M. Rs10887800 renalase gene polymorphism is associated with an increased risk of coronary artery disease in hemodialyzed patients. Int Urol Nephrol. 2016; 48(6): 871-876 doi: 10.1007/s11255-016-1270-7, indexed in Pubmed: 27023477.

19. Dickson ME, Sigmund CD. Genetic basis of hypertension: revisiting angiotensinogen. Hypertension. 2006; 48(1): 14-20, doi: 10.1161/01. HYP.0000227932.13687.60, indexed in Pubmed: 16754793

20. Banaszak B, Świetochowska E, Banaszak P, et al. Endothelin-1 (ET-1), $\mathrm{N}$-terminal fragment of pro-atrial natriuretic peptide (NTpro-ANP), and tumour necrosis factor alpha (TNF- $\alpha$ ) in children with primary hypertension and hypertension of renal origin. Endokrynol Pol. 2019; 70(1): 37-42, doi: 10.5603/EP.a2018.0079, indexed in Pubmed: 30359461.

21. Krysiak R, Sierant M, Marek B, et al. The effect of angiotensin-converting enzyme inhibitors on plasma adipokine levels in normotensive patients with coronary artery disease. Endokrynol Pol. 2010; 61(3): 280-287, indexed in Pubmed: 20602303

22. Maciorkowska D, Zbroch E, Malyszko J. Circulating renalase, catecholamines, and vascular adhesion protein 1 in hypertensive patients. J Am Soc Hypertens. 2015; 9(11): 855-864, doi: 10.1016/j.jash.2015.08.002, indexed in Pubmed: 26403854.

23. Wybraniec MT, Mizia-Stec K, Trojnarska O, et al. Low plasma renalase concentration in hypertensive patients after surgical repair of coarctation of aorta. J Am Soc Hypertens. 2014; 8(7): 464-474, doi: 10.1016/j. jash.2014.04.009, indexed in Pubmed: 25064768

24. Schlaich M, Socratous F, Eikelis N, et al. Renalase plasma levels are associated with systolic blood pressure in patients with resistant hypertension J Hypertens. 2010; 28: e437, doi: 10.1097/01.hjh.0000379519.82971.02.

25. Zhao Qi, Fan Z, He J, et al. Renalase gene is a novel susceptibility gene for essential hypertension: a two-stage association study in northern Han Chinese population. J Mol Med (Berl). 2007; 85(8): 877-885, doi: 10.1007/s00109-006-0151-4, indexed in Pubmed: 17216203.

26. Elsetohy KA, Al-Ghussein MA, Sabry D, et al. Are renalase rs2576178 and rs10887800 polymorphisms associated with pregnancy induced hypertension? World J Pharm Sci. 2014; 3(8): 177-192.

27. Bagci B, Karakus S, Bagci G, et al. Renalase gene polymorphism is associated with increased blood pressure in preeclampsia. Pregnancy Hypertens. 2016; 6(2): 115-120, doi: 10.1016/j.preghy.2016.04.002, indexed in Pubmed: 27155338.

28. Teimoori B, Moradi-Shahrebabak M, Rezaei M, et al. Renalase rs10887800 polymorphism is associated with severe pre-eclampsia in southeast Iranian women. J Cell Biochem. 2019; 120(3): 3277-3285, doi: 10.1002/jcb.27595, indexed in Pubmed: 30304564.

29. Jamil Z, Shahid S, Baig E, et al. Serum anti mullerian hormone and renalase levels in predicting the risk of preeclampsia. Taiwan J Obstet Gynecol. 2019; 58(2): 188-191, doi: 10.1016/j.tjog.2019.01.003, indexed in Pubmed: 30910136

30. Niadany SEl, Gayed AEl, Gayed EEl. Renalase rs10887800 gene polymorphism and its serum level in preeclampsia. Meta Gene. 2020; 24: 2214, doi: 10.1016/j.mgene.2020.100649.

31. Yılmaz ZV, Akkaş E, Yıldırım T, et al. A novel marker in pregnan with preeclampsia: renalase. J Matern Fetal Neonatal Med. 2017; 30(7): 808-813, doi: 10.1080/14767058.2016.1186637, indexed in Pubmed: 27147460

32. Zbroch E, Malyszko J, Malyszko JS, et al. Renalase, a novel enzyme involved in blood pressure regulation, is related to kidney function but not to blood pressure in hemodialysis patients. Kidney Blood Press Res. 2012; 35(6): 395-399, doi: 10.1159/000338178, indexed in Pubmed: 22539018

33. Zbroch E, Koc-Zorawska E, Malyszko J, et al. Circulating levels of renalase, norepinephrine, and dopamine in dialysis patients. Ren Fail 2013; 35(5): 673-679, doi: 10.3109/0886022X.2013.778754, indexed in Pubmed: 23530612.

34. Gluba-Brzózka A, Michalska-Kasiczak M, Franczyk-Skóra B, et al. Markers of increased cardiovascular risk in patients with chronic kidney disease. Lipids Health Dis. 2014; 13: 135, doi: 10.1186/1476-511X-13-135, indexed in Pubmed: 25145866.

35. Baek SHa, Cha RH, Kang SW, et al. Circulating renalase predicts all-cause mortality and renal outcomes in patients with advanced chronic kidney disease. Korean J Intern Med. 2019; 34(4): 858-866, doi: 10.3904/kjim.2017.058, indexed in Pubmed: 29172403.

36. Skrzypczyk P, Okarska-Napierała M, Stelmaszczyk-Emmel A, et al. Renalase in children with chronic kidney disease. Biomark ers. 2019; 24(7): 638-644, doi: 10.1080/1354750X.2019.1642957, indexed in Pubmed: 31293181.

37. Wiśniewska M, Serwin N, Dziedziejko V, et al. Chronic kidney disease is associated with increased levels of renalase in serum and decreased in erythrocytes. Pol Arch Intern Med. 2019; 129(11): 790-797, doi: 10.20452/pamw.15049, indexed in Pubmed: 31688840.

38. Pawlik A, Serdynska M, Dabrowska-Zamojcin E, et al. Renalase gene polymorphism in patients after renal allograft transplantation. Kidney Blood Press Res. 2014; 39(1): 58-64, doi: 10.1159/000355777, indexed in Pubmed: 24923329.

39. Zbroch E, Małyszko J, Małyszko J, et al. Renalase, kidney function, and markers of endothelial dysfunction in renal transplant recipients. Po Arch Med Wewn . 2012; 122(1-2): 40-44, doi: 10.20452/pamw.1132, indexed in Pubmed: 22237745.

40. Buraczynska M, Zukowski P, Buraczynska K, et al. Renalase gene polymorphisms in patients with type 2 diabetes, hypertension and stroke. Neuromolecular Med. 2011; 13(4): 321-327, doi: 10.1007/s12017-011-8158-6, indexed in Pubmed: 21964580.

41. Li X, Wang Z, Liu Y, et al. Association of imaging classification of intracranial cerebral atherosclerotic vascular stenosis in ischemic stroke and 
renalase gene polymorphisms. J Mol Neurosci. 2014; 52(4): 461-466, doi: 10.1007/s12031-013-0110-9, indexed in Pubmed: 24014100.

42. Zhang R, Li X, Liu N, et al. An association study on renalase polymorphisms and ischemic stroke in a Chinese population. Neuromolecular Med. 2013; 15(2): 396-404, doi: 10.1007/s12017-013-8227-0, indexed in Pubmed: 23564542.

43. Malyszko J, Koc-Zorawska E, Malyszko JS, et al. Renalase, stroke, and hypertension in hemodialyzed patients. Ren Fail. 2012; 34(6): 727-731, doi: 10.3109/0886022X.2012.681534, indexed in Pubmed: 22583169.

44. Reddy MV, Wang $\mathrm{H}$, Liu S, et al. Association between type 1 diabetes and GWAS SNPs in the southeast US Caucasian population. Genes Immun. 2011; 12(3): 208-212, doi: 10.1038/gene.2010.70, indexed in Pubmed: 21270831.

45. Wang F, Huang B, Li J, et al. Renalase might be associated with hypertension and insulin resistance in Type 2 diabetes. Ren Fail. 2014; 36(4): 552-556, doi: 10.3109/0886022X.2013.876352, indexed in Pubmed: 24742207.

46. Yatsenko SA, Rajkovic A. Genetics of human female infertilitył. Biol Reprod. 2019; 101(3): 549-566, doi: 10.1093/biolre/ioz084, indexed in Pubmed: 31077289.

47. Fatima SS, Rehman R, Martins RS, et al. Single nucleotide polymorphisms in Renalase and KCNQ1 genes and female infertility: A cross-sectional study in Pakistan. Andrologia. 2019; 51(10): e13434, doi: 10.1111/and.13434, indexed in Pubmed: 31579970.

48. Catak Z, Kocdemir E, Ugur K, et al. A Novel Biomarker Renalase and Its Relationship with its Substrates in Schizophrenia. J Med Biochem. 2019; 38(3): 299-305, doi: 10.2478/jomb-2018-0031, indexed in Pubmed: 31156340 .

49. Skrzypczyk P, Przychodzień J, Mizerska-Wasiak M, et al. Renalase in Children with Glomerular Kidney Diseases. Adv Exp Med Biol. 2017; 1021: 81-92, doi: 10.1007/5584 2017 22, indexed in Pubmed: 28405891.

50. Taranta-Janusz K, Roszkowska R, Wasilewska A. Renalase Levels in Children with Solitary Functioning Kidney. Indian Pediatr. 2015; 52(12): 1047-1050, doi: 10.1007/s13312-015-0771-2, indexed in Pubmed: 26713989 .

51. Lemiesz M, Tenderenda-Banasiuk E, Sosnowska D, et al. Serum Renalase Levels in Adolescents with Primary Hypertension. Pediatr
Cardiol. 2018; 39(6): 1258-1264, doi: 10.1007/s00246-018-1891-y, indexed in Pubmed: 29748702.

52. Rybi-Szumińska A, Michaluk-Skutnik J, Osipiuk-Remża B, et al. Normal values for urine renalase excretion in children. Pediatr Nephrol. 2014; 29(11): 2191-2195, doi: 10.1007/s00467-014-2855-y, indexed in Pubmed: 25060760.

53. Desir GV, Tang L, Wang P, et al. Renalase lowers ambulatory blood pressure by metabolizing circulating adrenaline. J Am Heart Assoc. 2012; 1(4): e002634, doi: 10.1161/JAHA.112.002634, indexed in Pubmed: 23130169.

54. Fedchenko V, Globa A, Buneeva O, et al. Renalase mRNA levels in the brain, heart, and kidneys of spontaneously hypertensive rats with moderate and high hypertension. Med Sci Monit Basic Res. 2013; 19: 267-270, doi: 10.12659/MSMBR.889540, indexed in Pubmed: 24113803.

55. $\mathrm{Wu} \mathrm{Y}, \mathrm{Xu} \mathrm{J}$, Velazquez $\mathrm{H}$, et al. Renalase deficiency aggravates ischemic myocardial damage. Kidney Int. 2011; 79(8): 853-860, doi: 10.1038/ki.2010.488, indexed in Pubmed: 21178975.

56. Lee HT, Kim JY, Kim M, et al. Renalase protects against ischemic AKI J Am Soc Nephrol. 2013; 24(3): 445-455, doi: 10.1681/ASN.2012090943, indexed in Pubmed: 23393318.

57. Zheng WL, Wang J, Mu JJ, et al. Effects of salt intake and potassium supplementation on renalase expression in the kidneys of Dahl salt-sensitive rats. Exp Biol Med (Maywood). 2016; 241(4): 382-386, doi: 10.1177/1535370215611584, indexed in Pubmed: 26553126.

58. Wang Y, Xie BO, Gao WH, et al. Effects of Renin-Angiotensin System Inhibitors on Renal Expression of Renalase in Sprague-Dawley Rats Fed With High Salt Diet. Kidney Blood Press Res. 2015; 40(6): 605-613, doi: 10.1159/000368536, indexed in Pubmed: 26619289.

59. Gu R, Lu W, Xie J, et al. Renalase deficiency in heart failure model of rats--a potential mechanism underlying circulating norepinephrine accumulation. PLoS One. 2011; 6(1): e14633, doi: 10.1371/journal. pone.0014633, indexed in Pubmed: 21297953.

60. Desir GV, Peixoto AJ. Renalase in hypertension and kidney disease. Nephrol Dial Transplant. 2014; 29(1): 22-28, doi: 10.1093/ndt/gft083, indexed in Pubmed: 24137013.

61. Malyszko J, Malyszko JS, Rysz J, et al. Renalase, hypertension, and kidney - the discussion continues. Angiology. 2013; 64(3): 181-187, doi: 10.1177/0003319712459212, indexed in Pubmed: 22969162 